\title{
Technical nuances of commonly used vascularised flaps for skull base reconstruction
}

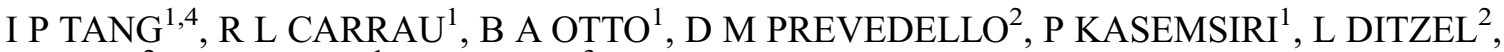 \\ $\mathrm{J} \mathrm{MUTO}^{2}, \mathrm{~B}_{\mathrm{KAPUCU}}{ }^{1}, \mathrm{C} \mathrm{KIRSCH}^{3}$ \\ Departments of ${ }^{1}$ Otolaryngology - Head and Neck Surgery, ${ }^{2}$ Neurological Surgery and ${ }^{3}$ Radiology, Wexner \\ Medical Centre, Ohio State University, Columbus, Ohio, USA, and ${ }^{4}$ Department of Otorhinolaryngology - Head \\ and Neck Surgery, Faculty of Medicine at the University Malaysia Sarawak, Malaysia
}

\begin{abstract}
Background and Methods: Reconstruction with a vascularised flap provides the most reliable outcome, with postoperative cerebrospinal fluid leak rates of less than 5 per cent. This article aims to review and summarise the critical technical aspects of the vascularised flaps most commonly used for skull base reconstruction.

Results: Vascularised flaps are classified as intranasal or extranasal. The intranasal group includes the Hadad-Bassagaisteguy nasoseptal flap, the Caicedo reverse nasoseptal flap, the nasoseptal rescue flap, the posteriorly or anteriorly based lateral wall flaps, and the middle turbinate flap. Extranasal flaps include the transfrontal pericranial and transpterygoid temporoparietal flaps.

Conclusion: The Hadad-Bassagaisteguy nasoseptal flap is overwhelmingly favoured for reconstructing extensive defects of anterior, middle and posterior cranial base. Its pertinent technical features are described. However, it is essential to master the skills required for the various extranasal or regional vascularised flaps because each can offer a reconstructive alternative for specific patients, especially when open approaches are needed and/or intranasal vascularised flaps are not feasible.
\end{abstract}

Key words: Skull Base; Reconstructive Surgical Procedures; Surgical Flaps; Pedicled Flaps

\section{Introduction}

The expansion of endoscopic endonasal approaches for resecting benign or malignant skull base tumours has led to improvements in the techniques available for reconstructing skull base defects. Developments in the approach, resection and reconstructive phases of endoscopic endonasal skull base surgery are based on a better understanding of the endoscopic surgical anatomy and improved endoscopic surgery techniques. These developments have allowed the surgical management of more substantial and complex tumours, which in turn create larger skull base defects requiring the use of more sophisticated reconstructive techniques.

Currently, reconstruction with a vascularised pedicle flap provides the most reliable outcomes, with postoperative cerebrospinal fluid leak rates of less than 5 per cent. ${ }^{1}$ Commonly used skull base vascularised flaps include the Hadad-Bassagaisteguy nasoseptal flap, ${ }^{2,3}$ nasoseptal rescue and modified rescue flaps, ${ }^{4}$ reverse rotation flap (Caicedo flap), ${ }^{5,6}$ anterior pedicle lateral nasal wall flap (Hadad-Bassagaisteguy 2 flap), ${ }^{7}$ posterior pedicle lateral nasal wall flap (Carrau-Hadad flap) ${ }^{8}$ middle turbinate flap, ${ }^{9}$ transpterygoid temporoparietal fascia flap, ${ }^{10,11}$ and transfrontal pericranial flap. ${ }^{12-14}$ Temporoparietal fascia and pericranial flaps are regional or extranasal vascularised flaps, whereas all others are intranasal. It should be noted that multiple flaps or a combination of vascularised flaps and free tissue grafts may be needed to complete skull base reconstruction.

\section{Surgical techniques}

The eight most commonly used skull base vascularised flaps are described and their indications and surgical techniques are discussed.

\section{Pedicle nasoseptal flap}

The pedicle nasoseptal flap (or Hadad-Bassagaisteguy flap) was developed at the University of Rosario, Argentina, by Hadad, Bassagaisteguy and colleagues. $^{2,3}$ The flap consists of the mucoperiosteum and mucoperichondrium of the nasal septum; it is pedicled on the posterior nasoseptal artery, a branch of the posterior nasal artery. This versatile flap is ideal for reconstructing extensive defects of the anterior, middle, clival and parasellar skull base. 